\title{
Elastic bending solutions in the Hamiltonian system
}

\author{
Weixiang Zhang ${ }^{1, \text { a }}$, Li Chen ${ }^{2, b}$ \\ ${ }^{1}$ Nantong polytechnic college, Nantong 450052, PR China \\ ${ }^{2}$ School of Civil Engineering and Architecture, Henan University of Technology, \\ Zhengzhou 450052, PR China \\ aWeixiang_zh@163.com, bichen@163.com
}

Keywords: Hamiltonian system; cantilever-beam; eigenvector

Abstract. In this paper, we introduce the Hamiltonian system to study bending problems of elastic cantilever-beam. The variational method and the variable seperation method are employed to establish the dual governing equations. Due to the completeness property of the eigenvectors, the solution can be expressed by the linear combination of these eigenvectors, and the coefficients of the combination are determined by the boundary conditions. In the numerical results, stress distributions of bending deformation are discussed.

\section{Introduction}

The cantilever beam has been widely applied in modern engineering. Its deformation is mainly in the form of bending. The traditional calculation method always using semi-inverse method such as the displacement method and the stress method [1,2]. In the 1850s, Saint-Venant proposed the famous semi-inverse solution method. According to this method, to obtain the complete general solutions of the governing equations, one can firstly assume some appropriate deformations or stresses. Then substitute the assumptions into the governing equations to find the other solutions, and lastly check the correctness of the assumptions. Due to the applying of assumptions of deformation or stress, it is necessory to verify the correctness of the final results. The semi-inverse method method has at least three limitations: (1) the solution is not complete, and can only approximately satisfy the boundary conditions. For example, when the fixed end of the cantilever beam is subjected to displacement constraints, the stress concentration will occur when external force are imposed [3]. The semi-inverse method cannot be explained this kind of concentration. (2) the assumption is only applicable to simple mechanical models, and for complex boundary condition problem, it is difficult to give reasonable assumptions. (3) when discussing the governing equations and boundary conditions using the displacement method or force method, it will inevitably lead to high order partial differential equations.

Zhong developed the exact symplectic approach for elastic problems on the basis of the mathematical theory of symplectic geometry [4]. In contrast to the well known semi-inverse method, this approach takes original variables and their dual variables as the basic variables, and hence the difficulty of solving high order differential equations in the traditional methods is overcome. The symplectic approach can explain the approximation of Saint-Venant principle theoretically: the exact solution consists of two parts, the Saint-Venant solution and the local solution. The local solution decays with the distance from the boundary, which is termed by the Saint-Venant principle, and is often neglected. In recent years, the symplectic approach gains much attention, and has been applied successfully into various branches of applied mechanics.

In this paper, on the bisis of variable seperation method and the principle of minimum energy, we apply the Hamiltonian system method to discussed the bending problem of the elastic cantilever beam. By using this method, the stress distribution of bending problems are obtained numerically, and stress concentration due to displacement constraints are well expained. Since the displacement and stress components are used as the basic variables, the Hamiltonian system method can be employeed to various branches of engineering problems. 


\section{Solution method}

Consider a homogeneous viscoelastic strip plane-domain in the Cartesian coordinate $(x, z)$. The origin is located at the central point of the free end of the domain, and the width and the length are $l$ and $2 h$, respectively. The boundary conditions at the free end and the fix end are

$$
\begin{aligned}
& \int_{-h}^{h} x \sigma_{z} d x=M, \quad \int_{-h}^{h} \tau_{x z} d x=P \quad(z=0) \\
& w=0, \quad u=0 \quad(z=l)
\end{aligned}
$$

The strain energy density function can be described as

$$
L=\frac{E}{2\left(1-v^{2}\right)}\left[\left(\partial_{x} u\right)^{2}+w^{2}+2 v \otimes_{x} u\right]+\frac{E}{4(1+v)}\left(\imath \&+\partial_{x} w\right)
$$

According to the principle of minimum potential energy, the result of the variation is just the dual equation in Hamiltonian system

$$
\psi \boldsymbol{\alpha}=\mathbf{H} \psi
$$

where $\psi=\{w, u, \sigma, \tau\}^{T}, \mathbf{H}$ is the Hamiltonian operator metrix.

$$
\mathbf{H}=\left[\begin{array}{cccc}
0 & -\omega_{1} \partial_{x} & \omega_{2} & 0 \\
-\partial_{x} & 0 & 0 & \omega_{3} \\
0 & 0 & 0 & -\partial_{x} \\
0 & -\omega_{4} \partial_{x}^{2} & -\omega_{1} \partial_{x} & 0
\end{array}\right]
$$

in which $\omega_{1}=v, \omega_{2}=\left(1-v^{2}\right) / E, \omega_{3}=2(1+v) / E, \omega_{4}=E$. By adopting the separation variable method, the governing equation is replace by the dual equations of the Hamiltonian system

$$
\mathbf{H} \psi_{j}(x)=\kappa_{j} \psi_{j}(x)
$$

where $\kappa_{j}$ is an eigenvalue, and $\psi_{j}$ is its cooresponding eigenvactor. Based on the property of the Hamiltonian operator matrix $\mathrm{H}$, the inner product of the functional is defined as

$$
<\psi_{1}, \mathbf{J}, \psi_{2}>=\int_{-1}^{1} \psi_{1}^{T} \mathbf{J} \psi_{2} d x
$$

where $\psi_{1}$ and $\psi_{2}$ are arbitrary eigenvectors, $\mathbf{J}$ is a unit rotational matrix. It can be proved that if $\mu_{j}$ is an eigenvalue, $-\mu_{j}$ is an eigenvalue also. Except zero eigenvelue, non-zero eigenvelues can be divided into two groups: $\mu_{\alpha_{j}}: \operatorname{Re}\left(\mu_{\alpha}\right)<0$ or $\operatorname{Re}\left(\mu_{\alpha}\right)=0, \operatorname{Im}\left(\mu_{\alpha}\right)<0$ and $\mu_{\beta}=-\mu_{\alpha}$. There are adjoint symplectic relationships of the ortho-normalization between the eigenvectors, namely 


$$
\begin{aligned}
& <\psi_{\alpha_{i}}, \mathbf{J}, \psi_{\beta_{j}}>=1,<\psi_{\beta_{i}}, \mathbf{J}, \psi_{\alpha_{j}}>=-1 \\
& <\psi_{\alpha_{i}}, \mathbf{J}, \psi_{\alpha_{j}}>=<\psi_{\beta_{i}}, \mathbf{J}, \psi_{\beta_{j}}>=0
\end{aligned}
$$

Thus an arbitrary whole state vector $\psi_{c}$ can always be expanded by the linear combination of the eigenvectors, or

$$
\psi_{c}=\sum\left[b_{n} \psi_{\alpha_{n}}(x) e^{\mu_{\alpha_{n}} z}+c_{n} \psi_{\beta_{n}}(x) e^{\mu_{\beta_{n}} z}\right]
$$

where $b_{n}=<\psi_{c}^{T}, \mathbf{J}, \psi_{\beta_{j}}>, c_{n}=-<\psi_{c}^{T}, \mathbf{J}, \psi_{\alpha_{j}}>$. It can be proved that the space of eigensolutions is complete.

\section{The special solutions}

Due to the adjoint symplectic relationship of ortho-normalization between the eigensolutions, the inhomogeneous term $\mathbf{f}$ can be developed as

$$
\mathbf{f}=\sum\left[d_{n}^{*}(z) \psi_{\alpha_{n}}(x)+g_{n}^{*}(z) \psi_{\beta_{n}}(x)\right]
$$

The coefficients $d_{n}(z)=\left[\mathbf{f}, \mathbf{J}, \psi_{\beta_{n}}(x)\right], \quad g_{n}(z)=<\mathbf{f}, \mathbf{J}, \psi_{\alpha_{n}}(x)>$. Suppose $\psi_{p}$ is a special solution:

$$
\psi_{p}=\sum\left[D_{n}(z) \psi_{\alpha_{n}}(x)+G_{n}(z) \psi_{\beta_{n}}(x)\right]
$$

Therefore

$$
\mathscr{W}_{n}^{\alpha}(z)=\mu_{a_{n}} D_{n}(z)+d_{n}(z) ; \quad \hat{\sigma}_{n}^{\alpha}(z)=-\mu_{\beta_{n}} G_{n}(z)+g_{n}(z)
$$

The solutions of Eqs.(38) are

$$
D_{n}(z)=\int_{0}^{z} d_{n}(\xi) e^{\mu_{\alpha_{n}}(z-\xi)} d \xi ; \quad G_{n}(z)=\int_{0}^{z} g_{n}(\xi) e^{-\mu_{\alpha_{n}}(z-\xi)} d \xi
$$




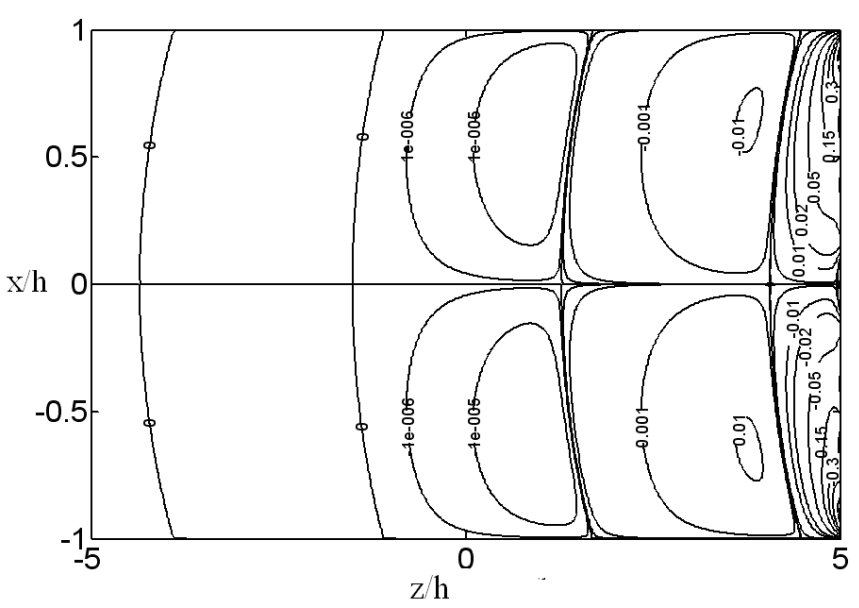

Fig. 1 The distribution of shear stress component $\tau / E$

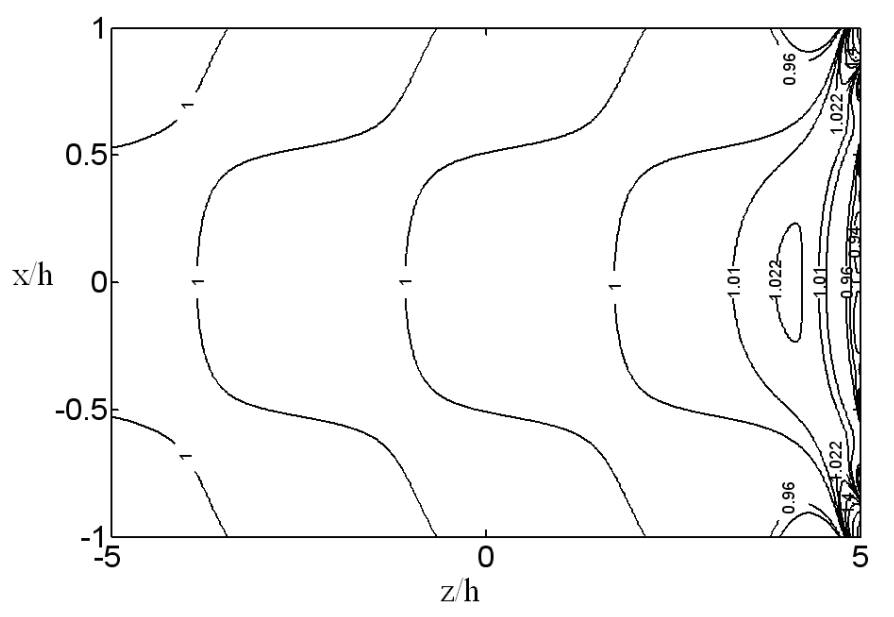

Fig. 2 The distribution of normal stress component $\sigma / E$

\section{Numerical computations}

Let's consider the bending problem when the free end is subjected to a simple couple $M$. The constants of the materials and the external couple are selected as: $E=4 G P a, v=0.25, h=0.1 \mathrm{~m}, l / h=5, M=4 K N$. Fig. 1 and Fig. 2 give contour lines of the normal stress and shear stress distributions. The figures explain that the stress concentrations are distinct near the boundary due to the restraints of the displacements. However, the concentrations fall off at a rapid rate along the coordinate $\mathrm{z}$. It can also be seen from the figures the maximum normal stress in this case are much smaller compared with the shear stresses. Thus, we can conclude that the shear stress should be considered firstly in the engineering practices.

\section{Conclusions}

Using the correspondence principle, The Hamiltonian system method is applied to the elastic materials bending problems, and all the eigenvectors obtained in analytical form. The eigenvector space is complete, and thus, the arbitrary boundary conditions expansion form of linear combination of eigenvectors, the combination coefficients can be determined according to the specific boundary conditions.

\section{References}

[1] L.J.De Chant: Comput. Math. Appl Vol. 43 (2002), p. 1161 
[2] Y. Huang, S.L. Croucht: Eng. Anal. Bound. Elem Vol. 29 (2013), p. 725

[3] X.S. Xu, W.X. Zhang, G.P. Wang, and X. Li: Int. J. Eng. Sci. Vol. 44 (2006), p. 897

[4] W.X. Zhong: Duality system in applied mechanics and optimal control. Kluwer Academic Publications, Norwich, NY (2004) 